\title{
ON THE CONTACT OZSVÁTH-SZABÓ INVARIANT
}

\author{
TOLGA ETGÜ AND BURAK OZBAGCI
}

\begin{abstract}
Sarkar and Wang proved that the hat version of Heegaard Floer homology group of a closed oriented 3-manifold is combinatorial starting from an arbitrary nice Heegaard diagram and in fact every closed oriented 3-manifold admits such a Heegaard diagram. Plamenevskaya showed that the contact Ozsváth-Szabó invariant is combinatorial once we are given an open book decomposition compatible with a contact structure. The idea is to combine the algorithm of Sarkar and Wang with the recent description of the contact Ozsváth-Szabó invariant due to Honda, Kazez and Matić. Here we simply observe that the hat version of the Heegaard Floer homology group and the contact Ozsváth-Szabó invariant in this group can be combinatorially calculated starting from a contact surgery diagram. We give detailed examples pointing out to some shortcuts in the computations.
\end{abstract}

\section{INTRODUCTION}

We know that every closed contact 3-manifold $(Y, \xi)$ can be obtained by a contact \pm 1 surgery on a Legendrian link in the standard contact $S^{3}([3])$. It is often convenient to describe $(Y, \xi)$ by a surgery diagram on the plane, i.e., by the projection of a Legendrian link in the standard contact $\left(\mathbb{R}^{3}, \operatorname{ker}(d z+x d y)\right)$ onto the $y z$-plane with a \pm 1 surgery coefficient assigned to each component of the link. Let $s_{\xi}$ denote the Spin ${ }^{c}$ structure induced by $\xi$. In order to calculate the Heegaard Floer homology group $\widehat{H F}\left(-Y, s_{\xi}\right)$ and in particular to identify the contact Ozsváth-Szabó invariant $c(\xi) \in \widehat{H F}\left(-Y, s_{\xi}\right)$ we first find a suitable open book decomposition compatible with $(Y, \xi)$ using the algorithm in [1] (see also [8],[17],[14],[18],[6],[7],[2]) and then construct a compatible Heegaard diagram for $-Y$ as in [9] which also includes a description of a certain cycle descending to $c(\xi)$ in $\widehat{H F}\left(-Y, s_{\xi}\right)$. Next we convert this Heegaard diagram into a nice Heegaard diagram ([16]) applying some finger moves without affecting the homology class $c(\xi)$ - no handle slides are necessary [15]. Finally we calculate $\widehat{H F}\left(-Y, s_{\xi}\right)$ and $c(\xi) \in \widehat{H F}\left(-Y, s_{\xi}\right)$ by simply counting certain squares and bigons in this nice Heegaard diagram. In fact this procedure will allow us to calculate $\widehat{H F}(-Y) \cong \widehat{H F}(Y)$, not just $\widehat{H F}\left(-Y, s_{\xi}\right)$.

2000 Mathematics Subject Classification. 57R17, 57R65, 57R58, 57M99.

Key words and phrases. Heegaard Floer homology, Ozsváth-Szabó invariants, contact structures, open book decomposition . 
We note that each step of the suggested combination of the above algorithms can be quite involved and one would like to reduce the calculations as much as possible by making certain choices. Here we demonstrate the significance of a particular choice in simplifying the calculations.

We assume that the reader is familiar with the basics of the Heegaard Floer theory (see [12], [13]). We will work with $\mathbb{Z}_{2}$ coefficients in our calculations throughout this paper.

\section{THE CONTACT OZSVÁTH-SZABÓ INVARIANT IS COMBINATORIAL}

Theorem 1. Let $(Y, \xi)$ be a closed contact 3-manifold described by a contact surgery diagram on the plane. We observe that the Spin ${ }^{c}$ structure $s_{\xi}$, Heegaard Floer homology groups $\widehat{H F}\left(-Y, s_{\xi}\right) \subseteq \widehat{H F}(Y)$ and the contact Ozsvath-Szabó invariant $c(\xi) \in$ $\widehat{H F}\left(-Y, s_{\xi}\right)$ can be calculated combinatorially.

Proof. Let $(Y, \xi)$ be a closed contact 3-manifold described by a contact surgery diagram on the plane, i.e., by the projection of a Legendrian link in the standard contact

$$
\left(\mathbb{R}^{3}, \operatorname{ker}(d z+x d y)\right) \subset\left(S^{3}, \xi_{s t}\right)
$$

onto the $y z$-plane with a \pm 1 surgery coefficient assigned to each component of the link. First we use the algorithm in [1] (see also [8],[17],[14],[18],[6],[7],[2]) to find an explicit open book decomposition compatible with $(Y, \xi)$. The idea in [1] is to embed the Legendrian surgery link into the pages of an open book decomposition in $S^{3}$ compatible with its standard contact structure and then perform the required contact surgeries to obtain an open book decomposition $\mathrm{OB}_{\xi}$ of $Y$ compatible with the resulting contact structure $\xi$.

Next we briefly recall ([9]) how to get a Heegaard diagram for $-Y$ which also includes a cycle that descends to the contact Ozsváth-Szabó invariant $c(\xi)$ starting from a given open book decomposition $\mathrm{OB}_{\xi}$ of $Y$ compatible with $\xi$. The open book decomposition $\mathrm{OB}_{\xi}$ can be described as follows: Let $S$ denote the page and let $h: S \rightarrow S$ denote the monodromy of $\mathrm{OB}_{\xi}$. Then $Y$ is homeomorphic to $S \times[0,1] / \sim$, where the equivalence relation is given by

$$
\begin{gathered}
(p, 1) \sim(h(p), 0), \quad p \in S \\
(p, t) \sim\left(p, t^{\prime}\right), \quad p \in \partial S ; t, t^{\prime} \in[0,1] .
\end{gathered}
$$

It is not too hard to see that $Y=H_{1} \cup H_{2}$ is a Heegaard splitting of $Y$, where $H_{1}=$ $S \times[0,1 / 2] / \sim$ and $H_{2}=S \times[1 / 2,1] / \sim$. Let $S_{0}$ and $S_{1 / 2}$ denote $S \times\{0\}$ and $S \times\{1 / 2\}$ in $H_{1}$, respectively. A basis on a compact surface $S$ with boundary is just a collection of properly embedded disjoint arcs $\left\{a_{1}, \ldots, a_{n}\right\}$ on $S$ such that when we cut $S$ along these arcs we get a single polygon. Now we choose a basis $\left\{a_{1}, \ldots, a_{n}\right\}$ on the page $S$ and choose a point $z$ in the polygonal region mentioned above. Consider the closed surface $\Sigma=S_{1 / 2} \cup-S_{0}$ and glue the arc $a_{i}$ on $S_{1 / 2}$ with the arc $a_{i}$ on $-S_{0}$ to obtain a closed curve 
$\alpha_{i}$ on $\Sigma$. Let $b_{i}$ be an arc which is isotopic to $a_{i}$ by a small isotopy so that the following hold:

(1) The endpoints of $a_{i}$ are isotoped along $\partial S_{1 / 2}$, in the direction given by the orientation of $S_{1 / 2}$.

(2) The arcs $a_{i}$ and $b_{i}$ intersect transversely in one point $x_{i}$ in the interior of $S_{1 / 2}$.

(3) If we orient $a_{i}$, and $b_{i}$ is given the induced orientation from the isotopy, then the sign of the intersection of $a_{i}$ and $b_{i}$ at $x_{i}$ is +1 .

Then consider the arc $h\left(b_{i}\right)$ on $-S_{0}$ and glue the arc $b_{i}$ and $h\left(b_{i}\right)$ to get a closed curve $\beta_{i}$ on $\Sigma$. If we let $\alpha=\left\{\alpha_{1}, \ldots, \alpha_{n}\right\}$ and $\beta=\left\{\beta_{1}, \ldots, \beta_{n}\right\}$, then $(\Sigma, \beta, \alpha, z)$ is a Heegaard diagram for $-Y$, while $(\Sigma, \alpha, \beta, z)$ is a Heegaard diagram for $Y$. Moreover $X=\left\{x_{1}, \ldots, x_{n}\right\} \in \mathbb{T}_{\alpha} \cap \mathbb{T}_{\beta} \subset \operatorname{Sym}^{n}(\Sigma)$ is a cycle in $\widehat{C F}(\Sigma, \beta, \alpha, z)$ which descends to the contact Ozsváth-Szabó invariant $c(\xi) \in \widehat{H F}(-Y)$, where $\mathbb{T}_{\alpha}=\alpha_{1} \times \cdots \times \alpha_{n}$ and $\mathbb{T}_{\beta}=\beta_{1} \times \cdots \times \beta_{n}$. Furthermore there is a map from the set of generators $\mathbb{T}_{\alpha} \cap \mathbb{T}_{\beta}$ of $\widehat{C F}(\Sigma, \beta, \alpha, z)$ to the set of $\operatorname{Spin}^{c}$ structures on $Y$. It turns out ([13]) that the special cycle $X$ corresponds to the $\operatorname{Spin}^{c}$ structure $s_{\xi}$ induced by $\xi$. Therefore $c(\xi)$ belongs to $\widehat{H F}\left(-Y, s_{\xi}\right) \subseteq \widehat{H F}(-Y)$. We note that $c_{1}(\xi)=c_{1}\left(s_{\xi}\right) \in H^{2}(Y ; \mathbb{Z})$ can be calculated ([4]) combinatorially from a given contact surgery diagram of $\xi$ (see page 195 in [11]).

A connected component of the complement of $\alpha$ and $\beta$ curves in $\Sigma$ is called a region. Now we use the algorithm of [16] to convert this Heegaard diagram into a nice Heegaard diagram, which we still denote by $(\Sigma, \beta, \alpha, z)$, so that all the regions on $\Sigma$ not including the base point $z$ are bigons and squares. In general we would need to apply finger moves and handle slides of the $\beta$ curves in the Heegaard diagram. Handle slides, fortunately, do not arise in our case [15] and a finger move corresponds to a certain kind of isotopy of the $\beta$ curves.

Recall that a domain is a formal linear combination of the regions on $\Sigma$. A domain $D$ is called an empty embedded $2 m$-gon, if

(1) $D$ has coefficients 0 and 1 everywhere,

(2) $D$ is topologically an embedded disk on $\Sigma$, with $2 m$ vertices on its boundary,

(3) There is exactly one region with coefficient 1 around each vertex on the $\partial D$,

(4) $D$ does not contain any intersection points of $\alpha$ and $\beta$ curves in its interior.

Once we have a nice Heegaard diagram $(\Sigma, \beta, \alpha, z)$, by [16], it is combinatorial to calculate the boundary map of the Heegaard Floer chain complex. We just make a list of all the generators and count all the empty embedded bigons and the empty embedded squares on the Heegaard surface connecting these generators by examining the diagram. Finally by using simple linear algebra with $\mathbb{Z}_{2}$ coefficients we can compute $\widehat{H F}(-Y)$. Here we emphasize that we can combinatorially determine all the generators which are mapped to the distinguished $\operatorname{Spin}^{c}$ structure $s_{\xi}$, calculate $\widehat{H F}\left(-Y, s_{\xi}\right)$ and identify $c(\xi) \in \widehat{H F}\left(-Y, s_{\xi}\right)$. 


\section{The Unique tight CONTACT STRUCtURE ON $S^{1} \times S^{2}$}

Consider the contact 3-manifold $(Y, \xi)$ described by the surgery diagram depicted in Figure 1, When we convert this diagram into a smooth diagram (cf. Figure 2) and blow down the -1-curve, we immediately see that the underlying 3-manifold $Y$ is nothing but $S^{1} \times S^{2}$. It is well-known that there exists a unique tight contact structure on $S^{1} \times S^{2}$ up to isotopy [5].

Proposition 2. The contact structure $\xi$ is the unique tight contact structure on $S^{1} \times S^{2}$.

Proof. Below we show that the contact Ozsváth-Szabó invariant $c(\xi)$ is nontrivial. Therefore by a fundamental result in [13] $\xi$ is tight.

Remark 3. In particular, the unique tight contact structure on $S^{1} \times S^{2}$ has nontrivial contact Ozsváth-Szabó invariant. This was first proved in [10].

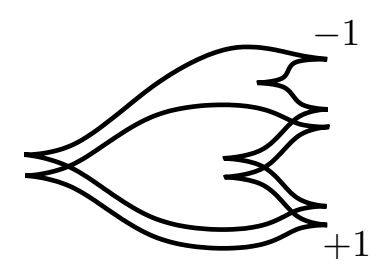

FIGURE 1. A contact surgery diagram
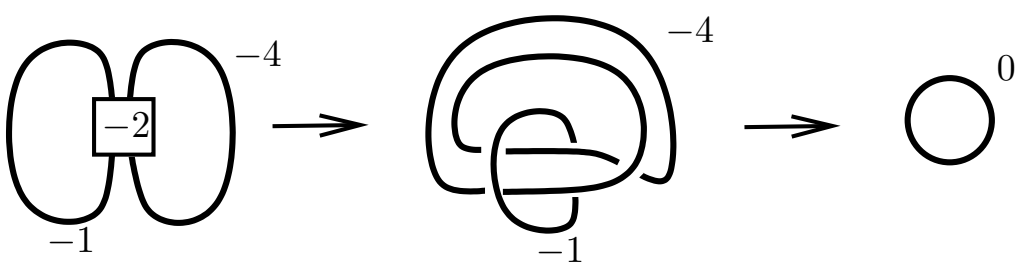

FIGURE 2. Underlying 3-manifold is $S^{1} \times S^{2}$

First we would like to understand the homotopy class of $\xi$ considered as an oriented plane field and determine the $\operatorname{Spin}^{c}$ structure $s_{\xi}$ induced by $\xi$. We calculate the first Chern class of $\xi$ as follows: Let $K_{1}$ and $K_{2}$ denote the \pm 1 surgery curves in Figure 1, respectively. Orient these Legendrian knots and let $\mu_{1}$ and $\mu_{2}$ denote the oriented meridians of $K_{1}$ and $K_{2}$, respectively. Then by [4] we have

$$
P D\left(c_{1}(\xi)\right)=\operatorname{rot}\left(K_{1}\right)\left[\mu_{1}\right]+\operatorname{rot}\left(K_{2}\right)\left[\mu_{2}\right]=\left[\mu_{1}\right]+2\left[\mu_{2}\right]=0 \in H_{1}\left(S^{1} \times S^{2}, \mathbb{Z}\right)
$$

where $P D$ denotes the Poincare dual and $\operatorname{rot}\left(K_{i}\right)$ denotes the rotation number of $K_{i}$. Moreover since $H_{1}\left(S^{1} \times S^{2} ; \mathbb{Z}\right)=\mathbb{Z}$ has no 2-torsion the $\operatorname{Spin}^{c}$ structure $s_{\xi}$ is determined by 
$c_{1}(\xi)$. In other words $s_{\xi}$ is the unique $\operatorname{Spin}^{c}$ structure on $S^{1} \times S^{2}$ whose first Chern class is trivial.

Our goal, however, is to calculate $\widehat{H F}\left(-Y, s_{\xi}\right), \widehat{H F}(-Y)$ and in particular the contact Ozsváth-Szabó invariant $c(\xi) \in \widehat{H F}\left(-Y, s_{\xi}\right)$. By applying the techniques in [6] we can find an open book decomposition $\mathrm{OB}_{\xi}$ (see Figure 8) compatible with $\xi$ : First we start with the open book decomposition $\mathrm{OB}_{H}$ induced by the positive Hopf link $H$ in $S^{3}$, whose page is an annulus. Then we stabilize this open book decomposition once and embed the +1 surgery curve onto a page. Next we stabilize one more time and embed the -1 surgery curve onto a page. Applying the required surgeries we get the desired open book decomposition. Note that we get exactly the same open book considered by Plamenevskaya in [15]. By the lantern relation on the four punctured sphere we know that the monodromy of $\mathrm{OB}_{\xi}$ is a product of two right-handed Dehn twists and hence $\xi$ is Stein fillable [8]. Therefore we know that the contact Ozsváth-Szabó invariant of $\xi$ is nontrivial [13]. In the following we will verify this fact by the algorithm described in [15], but we will choose three different bases to illustrate that this choice is in fact crucial in calculations.
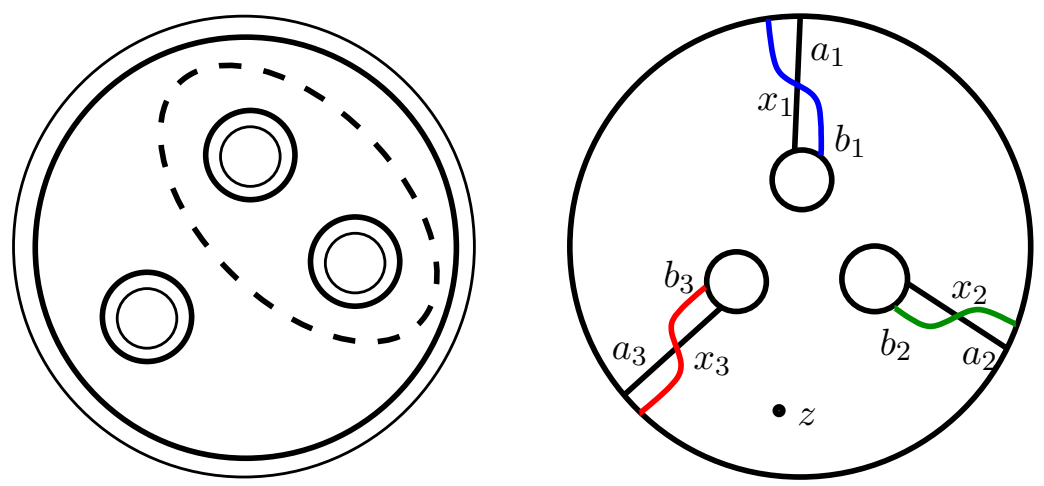

FIGURE 3. Left: Dehn twists about the solid curves are right-handed, while the Dehn twist about the dashed curve is left-handed. Right: A basis $\left\{a_{1}, a_{2}, a_{3}\right\}$ on the page $S_{1 / 2}$, the arcs $\left\{b_{1}, b_{2}, b_{3}\right\}$, the intersection points $\left\{x_{1}, x_{2}, x_{3}\right\}$, and the base point $z$.

2.1. Basis I. First we take the basis $\left\{a_{1}, a_{2}, a_{3}\right\}$ on page $S$ which is shown on the right in Figure 3. This is the basis that was used in [15]. We observe that there are two "bad" regions, one non-disk the other a hexagon. We divide each of these regions into two square regions by a simple finger move ([15]) introducing a bigon in the process. The resulting curves are depicted in Figure 5. After this modification of the Heegaard diagram there are nine regions which do not contain $z$. These regions are denoted by $R_{1}, \ldots, R_{9}$ and labelled by their indices in Figure 5 . 


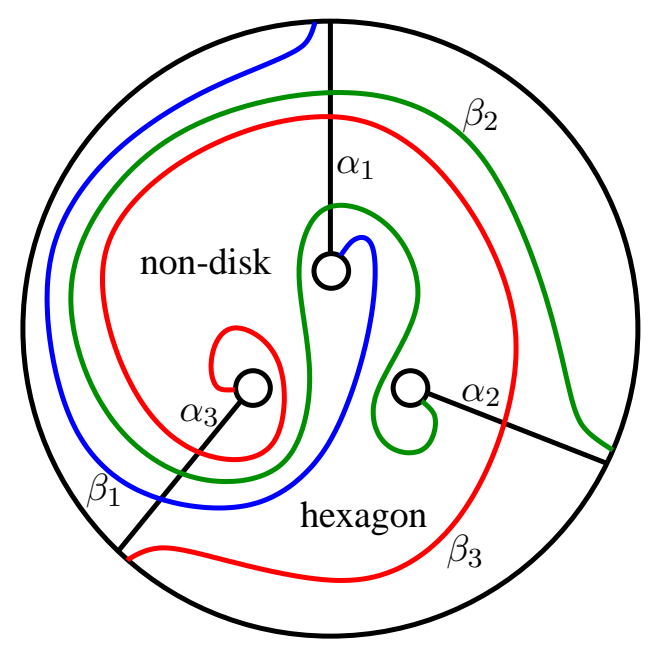

FIGURE 4. Bad regions are indicated on page $S_{0}$

Now by examining the intersections of $\alpha$ and $\beta$ curves on $\Sigma$ we see that the generators of the Heegaard Floer chain complex $\widehat{C F}(\Sigma, \beta, \alpha, z)$ are $X=\left(x_{1}, x_{2}, x_{3}\right), A=$ $\left(x_{1}, z_{1}, v_{3}\right), B=\left(x_{1}, x_{2}, z_{3}\right), C_{k}=\left(y_{k}, x_{2}, z_{2}\right), D_{i j}=\left(v_{i}, w_{j}, z_{3}\right), E_{i j}=\left(v_{i}, w_{j}, x_{3}\right), F_{i}=$ $\left(v_{i}, z_{1}, z_{2}\right), G_{k j}=\left(y_{k}, w_{j}, v_{3}\right)$, where $1 \leq i, j \leq 2$ and $1 \leq k \leq 3$. We calculated all the boundary maps induced by the empty embedded bigons and empty embedded squares:

$$
\begin{aligned}
& \partial X=0 \\
& \partial A=B \text { by } R_{3}+R_{4} \\
& \partial B=0 \\
& \partial C_{1}=0 \\
& \partial C_{2}=X+C_{1} \text { by } R_{4}+R_{7}+R_{8} \text { and } R_{9} \\
& \partial C_{3}=B \text { by } R_{4}+R_{8} \\
& \partial D_{11}=B+G_{11}+D_{12} \text { by } R_{1}, R_{5} \text { and } R_{6} \\
& \partial D_{12}=G_{12} \text { by } R_{5} \\
& \partial D_{21}=D_{22} \text { by } R_{6} \\
& \partial D_{22}=0 \\
& \partial E_{11}=X+E_{12} \text { by } R_{1} \text { and } R_{6} \\
& \partial E_{12}=0 \\
& \partial E_{21}=E_{22} \text { by } R_{6} \\
& \partial E_{22}=0 \\
& \partial F_{1}=E_{12}+C_{1} \text { by } R_{2} \text { and } R_{3}+R_{4}+R_{5} \\
& \partial F_{2}=E_{22}+C_{3}+A \text { by } R_{2}, R_{3} \text { and } R_{8} \\
& \partial G_{11}=G_{12} \text { by } R_{6} \\
& \partial G_{12}=0
\end{aligned}
$$




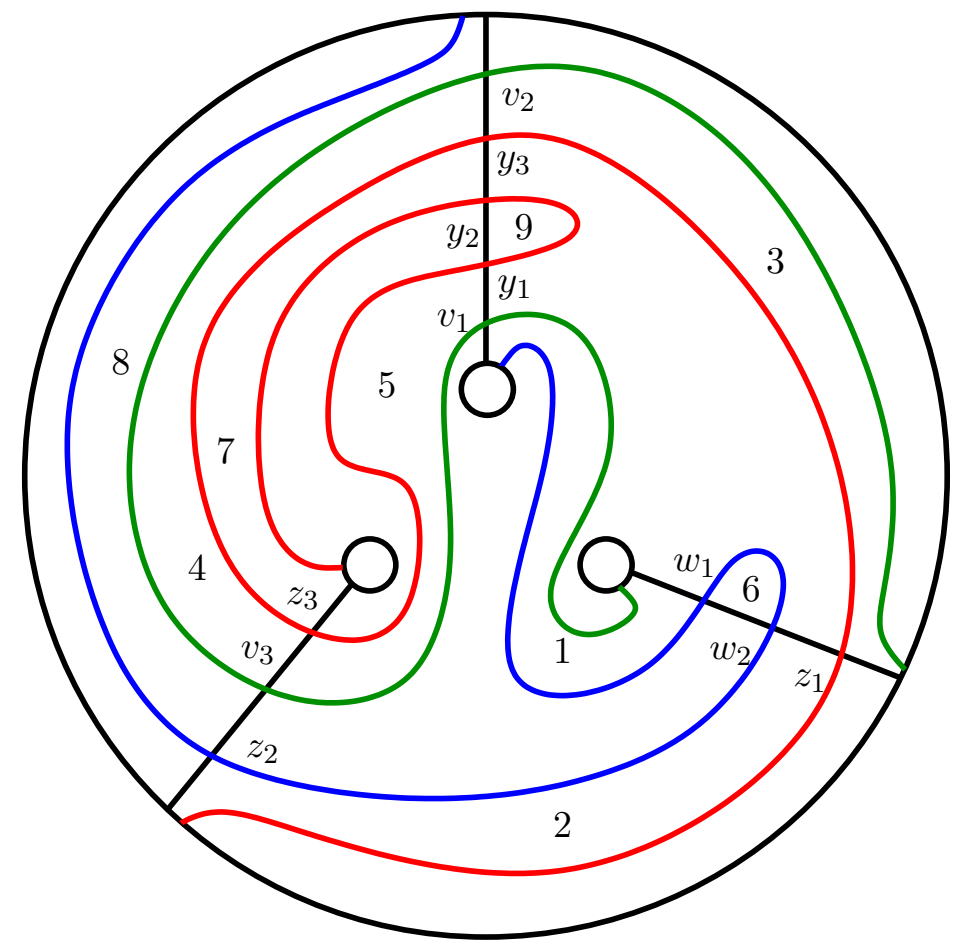

FIGURE 5. Finger moves

$\partial G_{21}=E_{21}+G_{11}+G_{22}$ by $R_{4}+R_{7}, R_{9}$ and $R_{6}$

$\partial G_{22}=E_{22}+G_{12}$ by $R_{4}+R_{7}$ and $R_{9}$

$\partial G_{31}=D_{21}+G_{32}$ by $R_{4}$ and $R_{6}$

$\partial G_{32}=D_{22}$ by $R_{4}$

The generators split into two sets: In the first set we have the generators $X, C_{1}, C_{2}$, $E_{11}, E_{12}, F_{1}$ with the following boundary maps: $\partial X=0, \partial C_{1}=0, \partial C_{2}=X+C_{1}$, $\partial E_{12}=0, \partial E_{11}=X+E_{12}, \partial F_{1}=E_{12}+C_{1}$. Note that these generators all correspond to the $\operatorname{Spin}^{c}$ structure $s_{\xi}$ because we know ([9]) that the cycle $X$ corresponds to $s_{\xi}$, and there are Whitney disks connecting $X$ and $C_{2}, C_{2}$ and $C_{1}, C_{1}$ and $F_{1}, F_{1}$ and $E_{12}, E_{12}$ and $E_{11}$. Similarly, there exist Whitney disks connecting the other 16 generators. Let $V_{1}$ be the vector space over $\mathbb{Z}_{2}$ generated by $X, C_{1}, C_{2}, E_{11}, E_{12}, F_{1}$ and let $\partial_{1}: V_{1} \rightarrow V_{1}$ denote the linear map induced by the boundary maps. Then it is easy to see that rank $\partial_{1}=2$ and dim ker $\partial_{1}=4$. It follows that $\widehat{H F}\left(-S^{1} \times S^{2}, s_{\xi}\right)=\mathbb{Z}_{2} \oplus \mathbb{Z}_{2}$ which is generated by $[X]=c(\xi)$ and $\left[C_{2}+E_{11}+F_{1}\right]$. Hence we conclude that $c(\xi) \neq 0$.

Remark 4. In [15], Plamenevskaya argues that $\partial E_{11}=X+E_{12}(d \boldsymbol{x}=\boldsymbol{c}+\boldsymbol{y}$ in her notation) is sufficient to show that $[X] \neq 0$. But in fact one has to show that $E_{12}$ is not 
a boundary. For a complete argument one has take into account the boundary relations $\partial F_{1}=E_{12}+C_{1}$ and $\partial C_{2}=X+C_{1}$.

To see that the generators in the second set correspond to a different Spin $^{c}$ structure $s \neq s_{\xi}$, consider the loop $\Gamma$ in the Heegaard surface $\Sigma$ obtained by concatenating the following paths: part of $\alpha_{1}$ from $x_{1}$ to $v_{2}$, part of $\beta_{2}$ from $v_{2}$ to $x_{2}$, part of $\alpha_{2}$ from $x_{2}$ to $z_{1}$, part of $\beta_{3}$ from $z_{1}$ to $x_{3}$, part of $\alpha_{3}$ from $x_{3}$ to $z_{2}$ and part of $\beta_{1}$ from $z_{2}$ to $x_{1}$. According to [12], the difference between the $\operatorname{Spin}^{c}$ structures which correspond to $X=\left(x_{1}, x_{2}, x_{3}\right)$ and $F_{2}=\left(v_{2}, z_{1}, z_{2}\right)$ is measured by the Poincaré dual of $p([\Gamma])$ in $H^{2}\left(S^{1} \times S^{2} ; \mathbb{Z}\right)$, where

$$
p: H_{1}(\Sigma ; \mathbb{Z}) \rightarrow \frac{H_{1}(\Sigma ; \mathbb{Z})}{<\left[\alpha_{1}\right],\left[\alpha_{2}\right],\left[\alpha_{3}\right],\left[\beta_{1}\right],\left[\beta_{2}\right],\left[\beta_{3}\right]>} \cong H_{1}\left(S^{1} \times S^{2} ; \mathbb{Z}\right) \cong \mathbb{Z}
$$

is the quotient homomorphism. In Figure 6, the curve $\Gamma$ is drawn on the Heegaard surface $\Sigma$ together with $\gamma_{i}$ 's such that $\left[\gamma_{i}\right]$ 's complete $\left[\alpha_{i}\right]$ 's to a basis for the first homology of $\Sigma$.

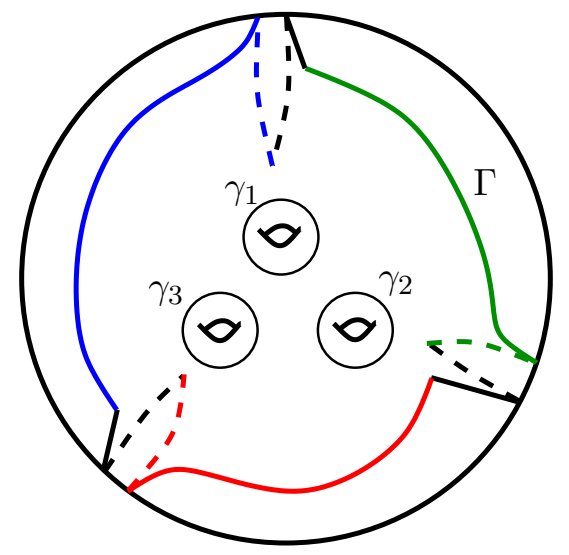

FIGURE 6. The curve $\Gamma$ on the Heegaard surface $\Sigma$

On one hand $[\Gamma]=\left[\gamma_{1}\right]+\left[\gamma_{2}\right]+\left[\gamma_{3}\right] \in H_{1}(\Sigma ; \mathbb{Z})$, where each of these curves is oriented "clockwise". On the other hand, the kernel of the quotient epimorphism $p$ is generated by $\left[\gamma_{1}\right]+\left[\gamma_{3}\right],\left[\gamma_{2}\right]+\left[\gamma_{3}\right]$ and $\left[\alpha_{i}\right]$ 's (see Figure 7). Therefore $p([\Gamma])$ is $\pm 1 \in \mathbb{Z} \cong H_{1}\left(S^{1} \times\right.$ $S^{2} ; \mathbb{Z}$ ), in particular nonzero. This implies that the generators $X$ and $F_{2}$ of the Heegaard Floer chain complex correspond to different $\operatorname{Spin}^{c}$ structures, i.e. $s \neq s_{\xi}$.

Let $V_{2}$ be the vector space generated by the remaining generators and let $\partial_{2}: V_{2} \rightarrow V_{2}$ denote the boundary map. One can calculate by simple linear algebra that rank $\partial_{2}=8$ and $\operatorname{dim} \operatorname{ker} \partial_{2}=8$. Hence we conclude that the homology for $\left(V_{2}, \partial_{2}\right)$ is trivial, i.e., $\widehat{H F}\left(-S^{1} \times S^{2}, s\right)=0$. Since there are no other generators, the Heegaard Floer homology groups in the other $\operatorname{Spin}^{c}$ structures are automatically zero. Consequently we get

$$
\widehat{H F}\left(-S^{1} \times S^{2}\right)=\widehat{H F}\left(-S^{1} \times S^{2}, s_{\xi}\right) \oplus \widehat{H F}\left(-S^{1} \times S^{2}, s\right)=\mathbb{Z}_{2} \oplus \mathbb{Z}_{2},
$$




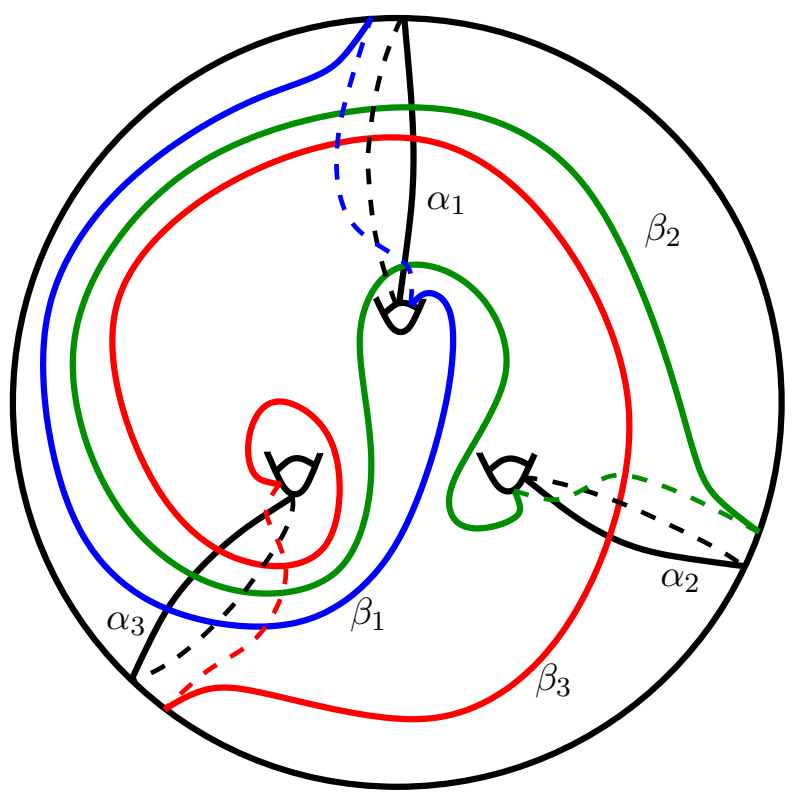

FIGURE 7. The $\alpha$ and $\beta$ curves on $\Sigma=S_{1 / 2} \cup-S_{0}$ pictured from the $S_{0}$-side.

which was indeed proved in [12].

2.2. Basis II. In the following we choose a different basis on the page $S$ of $\mathrm{OB}_{\xi}$ and repeat the calculations above. The point is that with this new choice of basis we will have fewer generators and fewer relations. We depict the $\alpha$ and $\beta$ curves on page $S_{1 / 2}$ in Figure 8 . Now by examining the intersections of $\alpha$ and $\beta$ curves on $\Sigma$ we see that there are exactly eight generators of the Heegaard Floer chain complex: $X=\left(x_{1}, x_{2}, x_{3}\right), A=\left(w_{2}, y_{1}, x_{3}\right), B=$ $\left(x_{1}, y_{2}, x_{3}\right), C=\left(x_{1}, z_{1}, y_{3}\right), D=\left(w_{2}, y_{3}, z_{2}\right), E=\left(w_{1}, x_{2}, z_{2}\right), F=\left(w_{1}, y_{2}, z_{2}\right), G=$ $\left(w_{1}, y_{1}, z_{1}\right)$.

There are five regions which do not contain $z$. These are denoted by $R_{1}, \ldots, R_{5}$ and labelled by their indices in Figure 9. Note that all of the regions are already squares. So we do not need to apply any finger moves. Below we list the boundary maps induced by these squares:

$$
\begin{aligned}
& \partial X=0 \\
& \partial A=B \text { by } R_{3} \\
& \partial B=0 \\
& \partial C=B \text { by } R_{5} \\
& \partial D=A+C \text { by } R_{3}+R_{4} \text { and } R_{4}+R_{5} \\
& \partial E=X+X=0 \text { by } R_{1} \text { and } R_{2}+R_{3}+R_{4}+R_{5} \\
& \partial F=B+D+G+B=D+G \text { by } R_{1}, R_{2}, R_{4} \text { and } R_{2}+R_{3}+R_{4}+R_{5}
\end{aligned}
$$



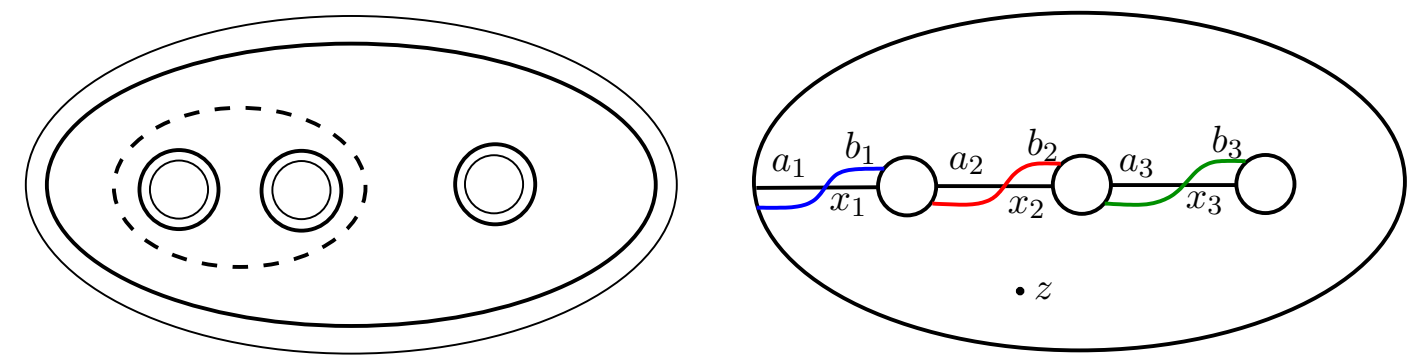

FIGURE 8. Left: Dehn twists about the solid curves are right-handed, while the Dehn twist about the dashed curve is left-handed. Right: A basis $\left\{\alpha_{1}, \alpha_{2}, \alpha_{3}\right\}$ on the page $S_{1 / 2}$, the arcs $\left\{b_{1}, b_{2}, b_{3}\right\}$, the intersection points $\left\{x_{1}, x_{2}, x_{3}\right\}$, and the base point $z$.

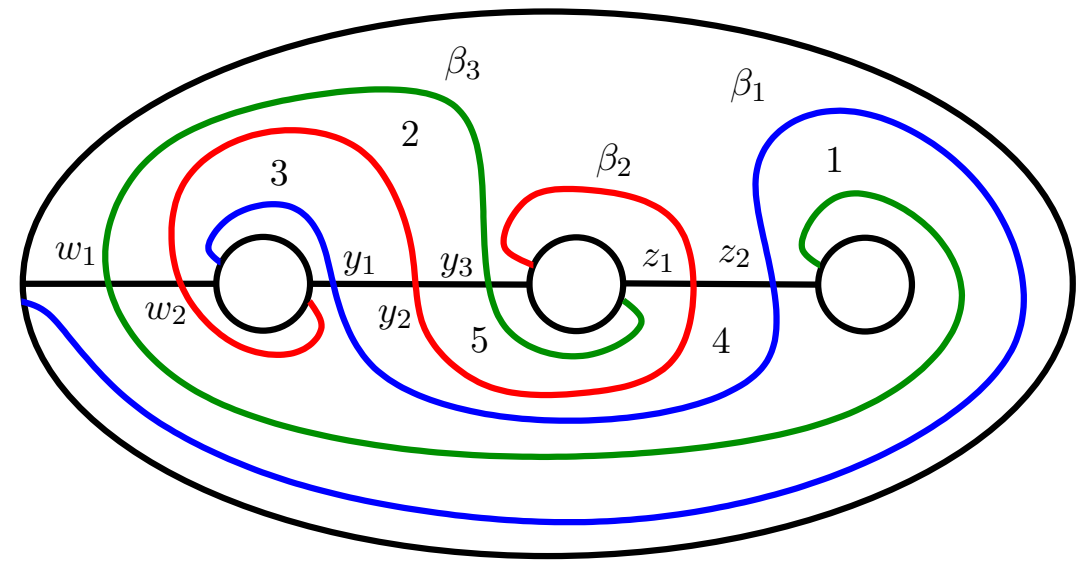

FIGURE 9. The $\alpha$ and $\beta$ curves on page $S_{0}$

$\partial G=A+C$ by $R_{2}+R_{3}$ and $R_{2}+R_{5}$

The chain complex naturally splits with respect to the $S p i n^{c}$ structures. The generators $X$ and $E$ correspond to the $\operatorname{Spin}^{c}$ structure $s_{\xi}$ which is uniquely determined by $c_{1}\left(s_{\xi}\right)=$ $c_{1}(\xi)=0$. The other generators correspond to a different $\operatorname{Spin}^{c}$ structure $s \neq s_{\xi}$. To see this consider the loop $\Gamma$ in the Heegaard surface $\Sigma$ obtained by concatenating the following paths: part of $\alpha_{1}$ from $x_{1}$ to $w_{1}$, part of $\beta_{3}$ from $w_{1}$ to $y_{3}$, part of $\alpha_{2}$ from $y_{3}$ to $x_{2}$, part of $\beta_{2}$ from $x_{2}$ to $z_{1}$, part of $\alpha_{3}$ from $z_{1}$ to $z_{2}$ and part of $\beta_{1}$ from $z_{2}$ to $x_{1}$. According to [12], the difference between the $\operatorname{Spin}^{c}$ structures which correspond to $C=\left(x_{1}, y_{3}, z_{1}\right)$ and $E=\left(w_{1}, x_{2}, z_{2}\right)$ is measured by the Poincaré dual of $p([\Gamma])$ in $H^{2}\left(S^{1} \times S^{2} ; \mathbb{Z}\right)$, where

$$
p: H_{1}(\Sigma ; \mathbb{Z}) \rightarrow \frac{H_{1}(\Sigma ; \mathbb{Z})}{<\left[\alpha_{1}\right],\left[\alpha_{2}\right],\left[\alpha_{3}\right],\left[\beta_{1}\right],\left[\beta_{2}\right],\left[\beta_{3}\right]>} \cong H_{1}\left(S^{1} \times S^{2} ; \mathbb{Z}\right) \cong \mathbb{Z}
$$


is the quotient homomorphism. In Figure 10, the curve $\Gamma$ is drawn on the Heegaard surface $\Sigma$ together with $\gamma_{i}$ 's such that $\left[\gamma_{i}\right]$ 's complete $\left[\alpha_{i}\right]$ 's to a basis for the first homology of $\Sigma$.

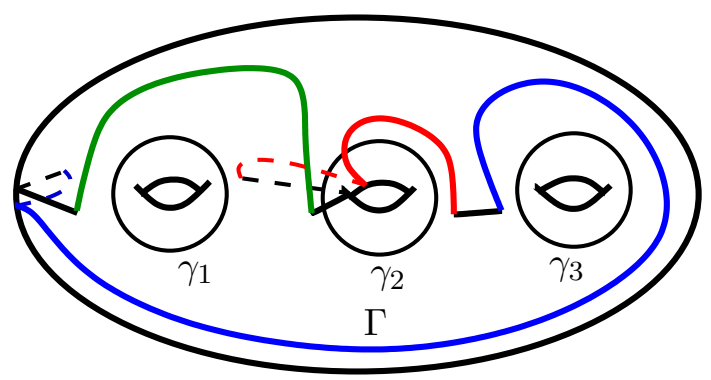

FIGURE 10. The curve $\Gamma$ on the Heegaard surface $\Sigma$

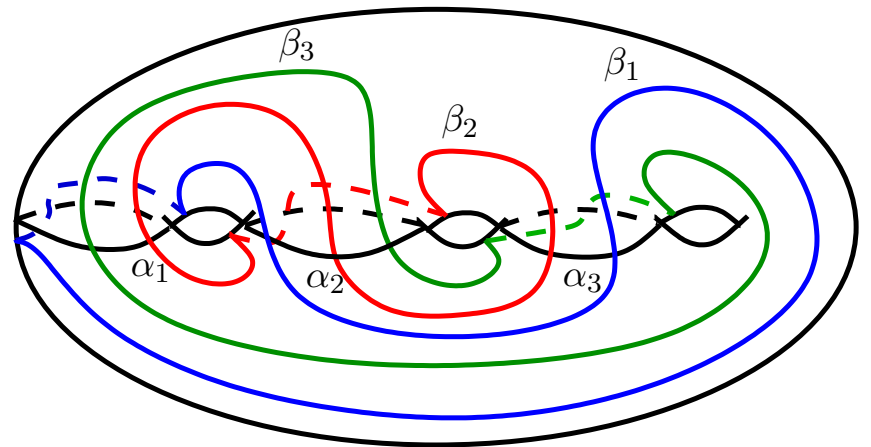

FIGURE 11. The $\alpha$ and $\beta$ curves on $\Sigma=S_{1 / 2} \cup-S_{0}$ pictured from the $S_{0}$-side.

On one hand $[\Gamma]=\left[\gamma_{1}\right]+\left[\gamma_{2}\right]+\left[\gamma_{3}\right] \in H_{1}(\Sigma ; \mathbb{Z})$, where each of these curves is oriented "clockwise". On the other hand, the kernel of the quotient epimorphism $p$ is generated by $\left[\gamma_{1}\right]+\left[\gamma_{3}\right],\left[\gamma_{1}\right]-\left[\gamma_{2}\right]$ and $\left[\alpha_{i}\right]$ 's (see Figure 11). Therefore $p([\Gamma])$ is $\pm 1 \in \mathbb{Z} \cong H_{1}\left(S^{1} \times\right.$ $\left.S^{2} ; \mathbb{Z}\right)$, in particular nonzero. This implies that the generators $C$ and $E$ of the Heegaard Floer chain complex correspond to different $\operatorname{Spin}^{c}$ structures, i.e. $s \neq s_{\xi}$. Moreover one can see that the homology induced by the generators $\{A, B, C, D, F, G\}$ is trivial. Therefore we conclude that $\widehat{H F}\left(-S^{1} \times S^{2}\right)=\widehat{H F}\left(-S^{1} \times S^{2}, s_{\xi}\right)=\mathbb{Z}_{2} \oplus \mathbb{Z}_{2}$ which is generated by $[X]$ and $[E]$. This confirms again that the contact class $[X]=c(\xi) \neq 0$.

2.3. Basis III. Interestingly there is yet another basis which simplifies the calculations dramatically. The basis given in Figure 12 produces only two generators $X=\left(x_{1}, x_{2}, x_{3}\right)$, and $A=\left(y_{1}, x_{2}, y_{3}\right)$. Other than the one which contains the base point $z$, there are four regions $R_{1}, \ldots, R_{4}$ indicated in Figure 13 by their indices and these regions are already 
squares. Moreover $\partial A=X+X=0$ by $R_{1}+R_{2}$ and $R_{3}+R_{4}$, and $\partial X=0$, confirming $\widehat{H F}\left(-S^{1} \times S^{2}\right)=\widehat{H F}\left(-S^{1} \times S^{2}, s_{\xi}\right)=\mathbb{Z}_{2} \oplus \mathbb{Z}_{2}$, the nontriviality of the contact class $[X]=c(\xi)$ and consequently the tightness of $\xi$.
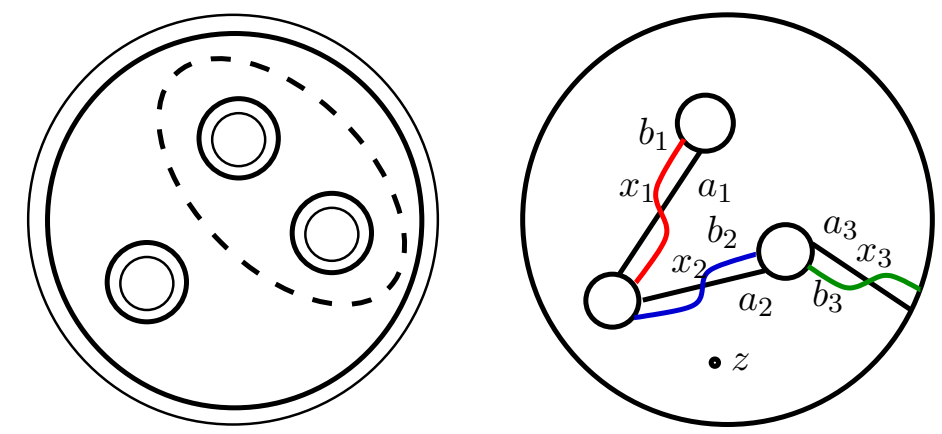

FIGURE 12. Left: Dehn twists about the solid curves are right-handed, while the Dehn twist about the dashed curve is left-handed. Right: A basis $\left\{a_{1}, a_{2}, a_{3}\right\}$ on the page $S_{1 / 2}$, the arcs $\left\{b_{1}, b_{2}, b_{3}\right\}$, the intersection points $\left\{x_{1}, x_{2}, x_{3}\right\}$, and the base point $z$.

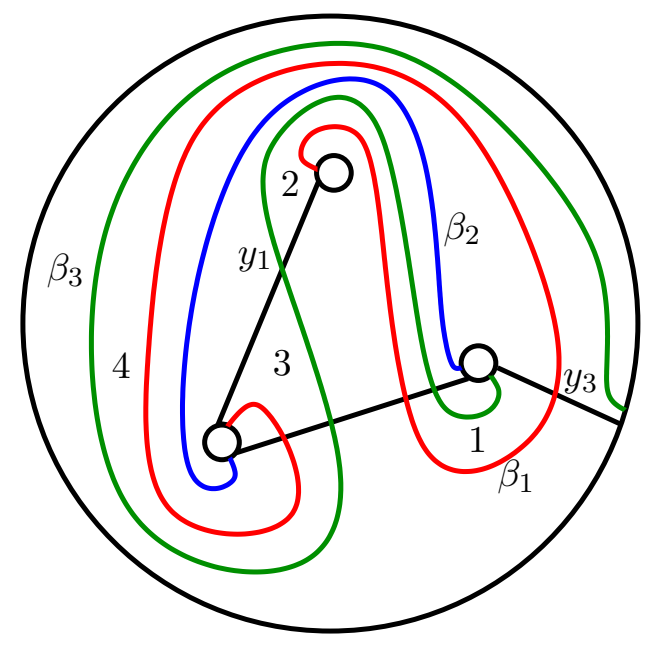

FIGURE 13.

\section{AN OVERTWISTED CONTACT STRUCTURE ON $S^{3}$}

Consider the contact 3-manifold $(Y, \xi)$ described by the surgery diagram depicted in Figure 14. When we convert this diagram into a smooth diagram (see Figure 15) and blow 
down the -1-curve, we immediately see that the underlying 3-manifold $Y$ is homeomorphic to $S^{3}$. Note that there is a unique $\operatorname{Spin}^{c}$ structure on $S^{3}$. From the contact surgery diagram we obtain an open book decomposition $O B_{\xi}$ on $S^{3}$ compatible with $\xi$ shown on the right of Figure 16 .

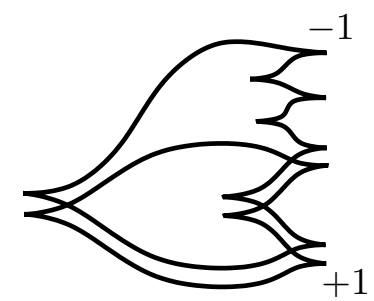

FIGURE 14. A contact surgery diagram

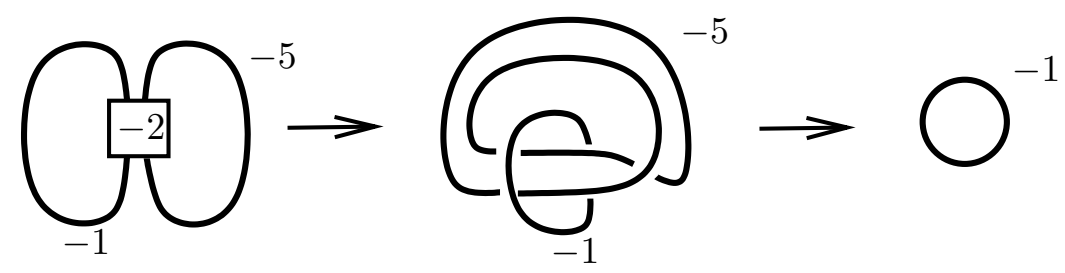

FIGURE 15. Underlying 3-manifold is $S^{3}$
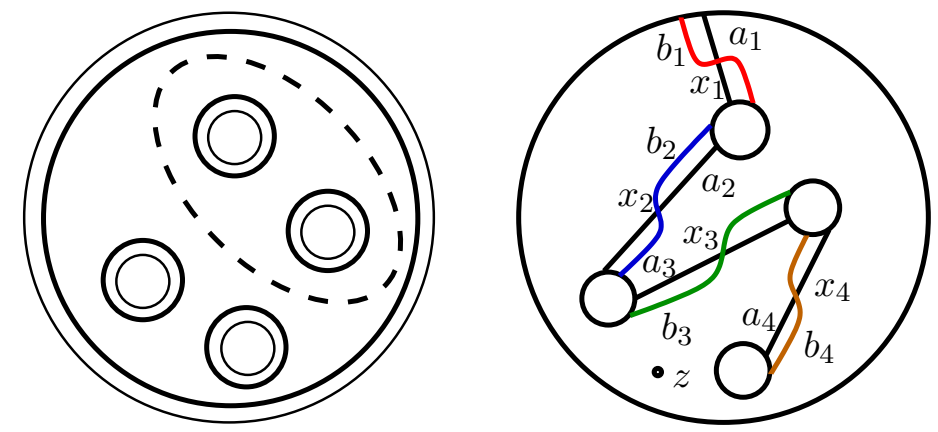

FIGURE 16. Left: Dehn twists about the solid curves are right-handed, while the Dehn twist about the dashed curve is left-handed. Right: A basis $\left\{a_{1}, a_{2}, a_{3}, a_{4}\right\}$ on the page $S_{1 / 2}$, the arcs $\left\{b_{1}, b_{2}, b_{3}, b_{4}\right\}$, the intersection points $\left\{x_{1}, x_{2}, x_{3}, x_{4}\right\}$, and the base point $z$.

Choosing a basis indicated on the right in Figure 16 gives the Heegaard diagram whose $\alpha$ and $\beta$ curves are shown in Figure 17. It is possible to convert this Heegaard diagram 


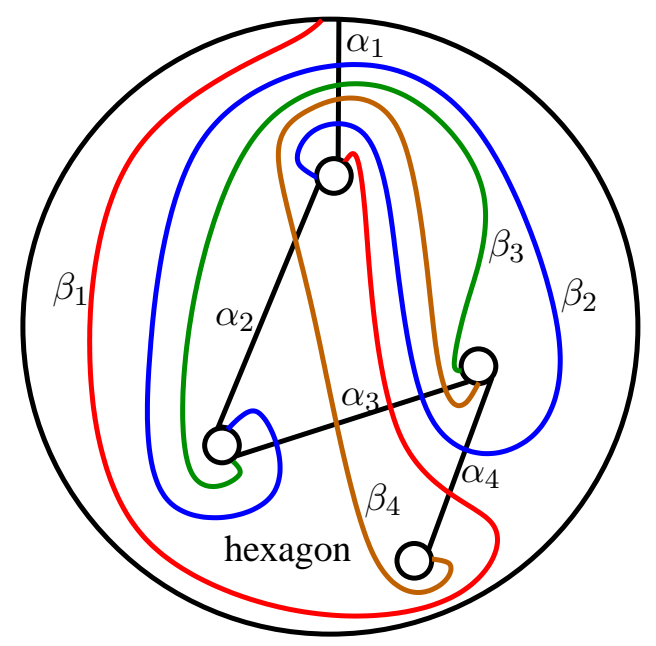

FIGURE 17. The bad region is indicated on page $S_{0}$

into one without any bad region (except for the region including the base point $z$ ), by a simple finger move. The curves of this new Heegaard diagram are depicted in Figure 18. There are 13 regions which do not contain $z$. These regions are denoted by $R_{1}, \ldots, R_{13}$ and labelled by their indices in Figure 18 . Examining the intersections of $\alpha$ and $\beta$ curves on the Heegaard surface $\Sigma$ one can confirm that the Heegaard Floer chain complex $\widehat{C F}(\Sigma, \beta, \alpha, z)$ have 29 generators in total: $X=\left(x_{1}, x_{2}, x_{3}\right), A=\left(x_{1}, r, x_{3}, n\right)$, $B_{i j}=\left(v_{i}, t_{j}, x_{3}, x_{4}\right), C_{i j}=\left(v_{i}, z_{j}, u, x_{4}\right), D_{i j}=\left(v_{i}, r, u, w_{j}\right), E_{i}=\left(v_{i}, r, x_{3}, p\right), F_{k}=$ $\left(y_{k}, x_{2}, u, x_{4}\right), G_{k}=\left(y_{k}, r, u, n\right), H_{i}=\left(q, t_{i}, x_{3}, n\right), I_{i}=\left(q, x_{2}, u, w_{i}\right), J=\left(q, x_{2}, x_{3}, p\right)$, $K_{i}=\left(q, z_{i}, u, n\right)$, where $1 \leq i, j, \leq 2$ and $1 \leq k \leq 3$. One can also calculate all the boundary maps:

$$
\begin{aligned}
& \partial X=0 \\
& \partial A=X \text { by } R_{9}+R_{11}+R_{12} \\
& \partial B_{11}=B_{21} \text { by } R_{4} \\
& \partial B_{12}=X+B_{22} \text { by } R_{1}+R_{2} \text { and } R_{4} \\
& \partial B_{2 j}=0 \\
& \partial C_{11}=B_{12}+C_{21}+F_{1} \text { by } R_{3}, R_{4} \text { and } R_{1} \\
& \partial C_{12}=B_{11}+C_{22} \text { by } R_{11}+R_{13} \text { and } R_{4} \\
& \partial C_{21}=B_{22}+F_{2} \text { by } R_{3} \text { and } R_{1}+R_{5} \\
& \partial C_{22}=B_{21} \text { by } R_{11}+R_{13} \\
& \partial D_{11}=D_{21} \text { by } R_{4} \\
& \partial D_{12}=C_{12}+D_{22}+E_{1} \text { by } R_{9}, R_{4} \text { and } R_{13} \\
& \partial D_{21}=0 \\
& \partial D_{22}=C_{22}+E_{2} \text { by } R_{9} \text { and } R_{13}
\end{aligned}
$$




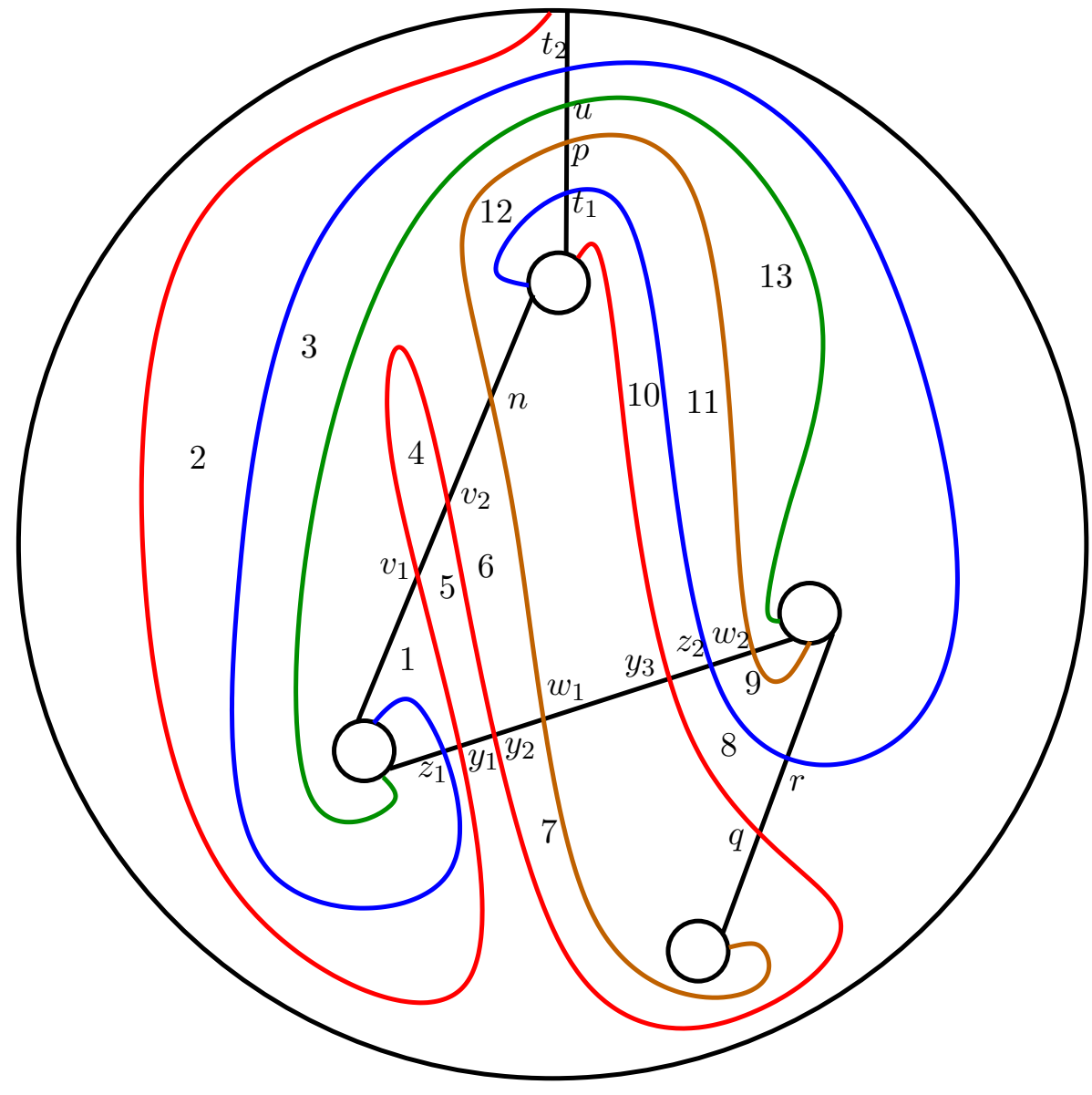

FIGURE 18. Finger move

$\partial E_{1}=B_{11}+E_{2}$ by $R_{9}+R_{11}$ and $R_{4}$

$\partial E_{2}=B_{21}$ by $R_{9}+R_{11}$

$\partial F_{1}=X+F_{2}$ by $R_{2}+R_{3}$ and $R_{4}+R_{5}$

$\partial F_{2}=0$

$\partial F_{3}=X$ by $R_{10}+R_{11}+R_{13}$

$\partial G_{1}=A+D_{11}+F_{1}+G_{2}$ by $R_{2}+R_{3}, R_{5}+R_{6}, R_{9}+R_{11}+R_{12}$ and $R_{4}+R_{5}$

$\partial G_{2}=D_{21}+F_{2}$ by $R_{6}$ and $R_{9}+R_{11}+R_{12}$

$\partial G_{3}=A+F_{3}$ by $R_{10}+R_{11}+R_{13}$ and $R_{9}+R_{11}+R_{12}$

$\partial H_{1}=A+B_{21}+J$ by $R_{8}+R_{10}, R_{6}+R_{7}$ and $R_{12}$

$\partial H_{2}=B_{22}$ by $R_{6}+R_{7}$

$\partial I_{1}=F_{2}$ by $R_{7}$

$\partial I_{2}=F_{3}+J$ by $R_{8}+R_{9}$ and $R_{13}$ 
$\partial J=X$ by $R_{8}+R_{9}+R_{10}+R_{11}$

$\partial K_{1}=C_{21}+H_{2}+I_{1}$ by $R_{6}+R_{7}, R_{3}$ and $R_{1}+R_{5}+R_{6}$

$\partial K_{2}=C_{22}+G_{3}+H_{1}+I_{2}$ by $R_{6}+R_{7}, R_{8}, R_{11}+R_{13}$ and $R_{11}+R_{12}$

From $\partial A=X$ one immediately sees that $c(\xi)=[X]=0 \in \widehat{H F}\left(-S^{3}, s_{\xi}\right)$ even with $\mathbb{Z}$ coefficients. By an important result in [13], $\xi$ is not Stein fillable. In fact, since the unique tight contact structure on $S^{3}$ is Stein fillable by [5], $\xi$ is overtwisted. On the other hand, it is seen that the image of the boundary map is 14 dimensional since it is generated by $\left\{X, B_{21}, B_{22}, D_{21}, F_{2}, A+F_{3}, A+J, B_{11}+C_{22}, B_{11}+E_{2}, B_{12}+C_{21}+F_{1}, A+D_{11}+\right.$ $\left.F_{1}+G_{2}, C_{12}+D_{22}+E_{1}, C_{21}+H_{2}+I_{1}, C_{22}+G_{3}+H_{1}+I_{2}\right\}$. Therefore the kernel is $29-14=15$ dimensional. Hence we verified that $\widehat{H F}\left(-S^{3}, s_{\xi}\right)=\widehat{H F}\left(-S^{3}\right)=\mathbb{Z}_{2}$.

Remark 5. Note that this contact structure has an open book decomposition which differs from the one in the previous section by an additional puncture and a right-handed Dehn twist around that puncture. It is interesting that these modifications, even though the Dehn twist is right-handed, convert a Stein fillable contact structure to an overtwisted one.

Remark 6. An alternative way to see the overtwistedness of the contact structure $\xi$ given by the contact surgery diagram in Figure 14 is to use the $d_{3}$ invariant of $\xi$ as a plane field and compare it with that of the unique tight contact structure on $S^{3}$. The former is $1 / 2$ whereas the latter is $-1 / 2$.

Acknowledgement: We would like to thank András Stipsicz for comments on a draft of this paper. TE was partially supported by a GEBIP grant of the Turkish Academy of Sciences and a CAREER grant of the Scientific and Technological Research Council of Turkey. BO was partially supported by a research grant of the Scientific and Technological Research Council of Turkey.

\section{REFERENCES}

[1] S. Akbulut and B. Ozbagci, Lefschetz fibrations on compact Stein surfaces, Geom. Topol. 5 (2001), 319-334.

[2] M. F. Arikan, On the support genus of a contact structure given by a surgery diagram, preprint, arXiv:math.GT/0704.1670

[3] F. Ding and H. Geiges, A Legendrian surgery presentation of contact 3-manifolds, Math. Proc. Cambridge Philos. Soc. 136 (2004), 583-598.

[4] F. Ding, H. Geiges and A. Stipsicz, Lutz twist and contact surgery, Asian J. Math. 9 (2005), no. 1, 57-64.

[5] Y. Eliashberg, Filling by holomorphic discs and its applications, Geometry of low-dimensional manifolds, 2 (Durham, 1989), 45-67, London Math. Soc. Lecture Note Ser., 151, Cambridge Univ. Press, Cambridge, 1990.

[6] J. B. Etnyre, Lectures on open book decompositions and contact structures, Floer homology, gauge theory, and low-dimensional topology, 103-141, Clay Math. Proc., 5, Amer. Math. Soc., Providence, RI, 2006. 
[7] J. B. Etnyre and B. Ozbagci, Open books and plumbings, Int. Math. Res. Notices vol. 2006, Article ID $72710,2006$.

[8] E. Giroux, Contact geometry: from dimension three to higher dimensions, Proceedings of the International Congress of Mathematicians (Beijing 2002), 405-414.

[9] K. Honda, W. Kazez and G. Matić, On the contact class in Heegaard Floer homology, preprint, arXiv:math.GT/0609734

[10] P. Lisca and A. I. Stipsicz, Seifert fibered contact three-manifolds via surgery, Algebr. Geom. Topol. 4 (2004), 199-217 (electronic).

[11] B. Ozbagci and A. I. Stipsicz, Surgery on contact 3-manifolds and Stein surfaces, Bolyai Soc. Math. Stud., Vol. 13, Springer, 2004.

[12] P. Ozsváth and Z. Szabó, Holomorphic disks and topological invariants for closed three-manifolds, Ann. of Math. (2) 159 (2004), no. 3, 1027-1158.

[13] P. Ozsváth and Z. Szabó, Heegaard Floer homology and contact structures, Duke Math. J. 129 (2005), no. 1, 39-61.

[14] O. Plamenevskaya, Contact structures with distinct Heegaard Floer invariants, Math. Res. Lett. 11 (2004), no. 4, 547-561.

[15] O. Plamenevskaya, A combinatorial description of the Heegaard Floer contact invariant, to appear in Algebr. Geom. Topol., arXiv:math.GT/0612322

[16] S. Sarkar and J. Wang, An algorithm for computing some Heegaard Floer homologies, preprint, arXiv:math.GT/0607777

[17] S. Schönenberger, Planar open books and symplectic fillings, PhD thesis, University of Pennsylvania, 2005.

[18] A. I. Stipsicz, Surgery diagrams and open book decompositions of contact 3-manifolds, Acta Math. Hungar. 108 (2005), no. 1-2, 71-86.

Department of Mathematics, Koç University, Istanbul, Turkey

E-mail address: tetgu@ku.edu.tr

E-mail address: bozbagci@ku.edu.tr 